\title{
Changing photospheric vector magnetic fields associated with a B4.2-class solar flare
}

\author{
Jiangtao $\mathbf{S u}^{1,2}, \mathbf{Y u} \mathbf{L i u}^{3}$ and Yuandeng $\mathrm{Shen}^{3}$ \\ ${ }^{1}$ Key Laboratory of Solar Activity, National Astronomical Observatories, \\ Chinese Academy of Science, Beijing 100012, China \\ email: sjt@bao.ac.cn \\ ${ }^{2}$ State Key Laboratory of Space Weather, Chinese \\ Academy of Sciences, Beijing 100190 \\ ${ }^{3}$ National Astronomical Observatories, Yunnan observatory, \\ Chinese Academy of Sciences
}

\begin{abstract}
Recent observations have provided evidence that the solar photospheric magnetic fields could have rapid and permanent changes in both longitudinal and transverse components associated with large (X- or M-class) solar flares. However, few observations have been reported about small flares. In this paper we find the observational evidence of changing photospheric vector magnetic fields associated with a B4.2-class flare obtained with the Solar Magnetic Field Telescope (SMFT) installed at Huairou Solar Observing Station (HSOS) of Nation Astronomical Observatories of Chinese Academy of Sciences.
\end{abstract}

Keywords. Sun: Photosphere; Sun: Flares; Sun: Magnetic fields

\section{Introduction}

On the Sun, due to the corona being $10^{-9}$ times as dense as the photosphere, it was usually believed that solar eruptions have no significant impact on the much denser photosphere. However, this prejudice changed gradually over the past 20 years with the accumulated observational evidence of changing photospheric magnetic fields associated with major solar flares being reported, e.g., changes of magnetic shear along the polarity inversion line (PIL) (Ambastha (1993), Wang (1992), and Li (2000)), rapid and permanent changes of longitudinal magnetic fields (Kosovichev \& Zharkova (2001) and Sudol \& Harvey (2005)), sudden and unbalanced magnetic flux changes (e.g., Spirock et al. (2002)), white-light (WL) structure changes in sunspots (e.g., Deng et al. (2005)), and an increase of transverse magnetic fields near the flaring PIL (e.g., Wang \& Liu (2010)). However, so far there is no a B-class flare event reported associated with changing magnetic fields. In this work, we report that such a small flare can also cause significant changes on photospheric magnetic fields

\section{Data analysis and results}

A B2.4 flare occurred at 04:18 UT in AR NOAA 8027 on 1997 April 9, which was caused by a tiny black feature (see in the WL image with negative polarity) invading into its surrounding positive polarity region. HSOS instrument well covered the whole erupting process in photosphere and chromosphere. The data obtained are vector magnetograms taken with $35 \mathrm{~cm}$ SMFT (the working line, FeI $5324.19 \AA$ ), and filtergrams of FeI 5324 $\AA$ and $\mathrm{H} \beta 4861.34 \AA$.

In Figure 1, panels (a) and (c) are the longitudinal fields before and after the flare, respectively, and (e) is the difference image of the pre- and post-flare longitudinal fields 

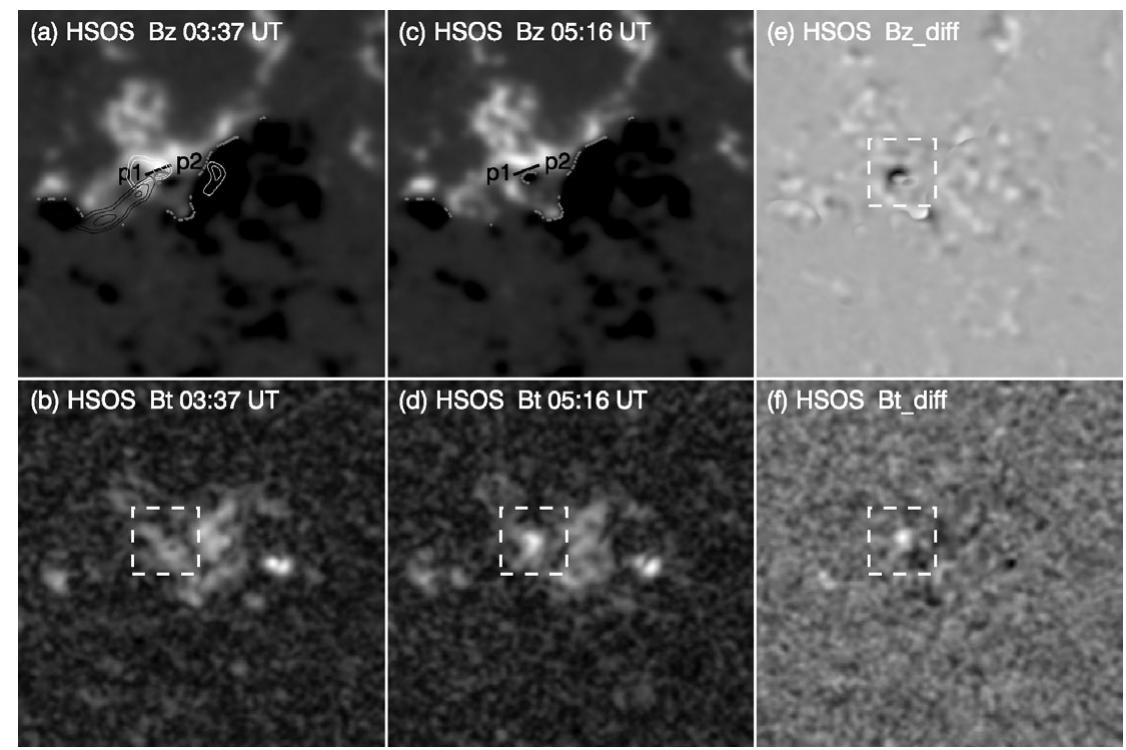

Figure 1. A comparison between pre-flare ( $a$ and b) and post-flare (c and d) states of the B4.2 flare on 1997 July 09. The panels (a and c) and (b and d) indicate HSOS longitudinal and transverse fields, respectively, and (e) and (f) are their corresponding difference images (the post-flare image minus the pre- flare one). The white and black contours in panel (a) represent HSOS $\mathrm{H} \beta$ flare and $\mathrm{H} \beta$ surge, respectively. The flaring PIL is highlighted by Line p1p2 shown in panels (a) and (c). The dashed rectangles in panels (b), (d), (e) and (f) are used as the position reference. The field of view $(\mathrm{FOV})$ is $105 \times 105$ arcsec.

(unsigned value); the bottom panels are the same as the top ones, but for the transverse fields and (f) is their difference image (signed value). The gray broken lines in panels (a) and (c) are the PIL of this AR. The dashed rectangles in panels (b), (d), (e) and (f) are plotted as the reference position of around the flaring PIL. The white contours in panel (a) was $\mathrm{H} \beta$ flare and the black contours $\mathrm{H} \beta$ surge occurring along with the flare. Line p1p2 in panel (a/c) was the position near the flaring PIL, to the left of which we find the longitudinal field showed a decrease (black patch) near the flaring PIL in panel (e), and the transverse field showed an increase (white patch) in panel (f).

In Figure 2, panels (a) and (b) in top row are two FeI $5324.19 \AA$ filtergrams before and after the flare, respectively, in which a tiny faint dark feature appeared at the positions highlighted by the black arrows. This tiny dark feature was moving to the upper left. To study its motion, two regions surrounding this feature are selected and marked by the dotted rectangles in Panels (a) and (b). A temporal sequence of the close-up view of the longitudinal magnetograms of the dotted region is shown from right to left in the bottom row of Figure 2. The dark feature did not emerge in the FOV until 02:01 UT. From then on, it moved gradually towards the top left direction at a speed of 0.6 $\mathrm{km} \mathrm{s}^{-1}$. Due to its continuous moving and colliding with the opposite polarity patches nearby, the magnetic reconnection unavoidably occurred, and produced this B4.2 flare as well as the surge (white and black contours in Panel (a) of Figure 1, respectively). panel (c) is the filtergram difference image, in which the white and black contours show the decayed (bright) and enhanced (dark) areas of intensity. Similar to X-class flares studied in previous studies (Liu et al. (2005)), the enhanced area (white contour) in this B-class flare is also surrounded by the decayed areas (black contours) which indicated the magnetic field changes in this area. 


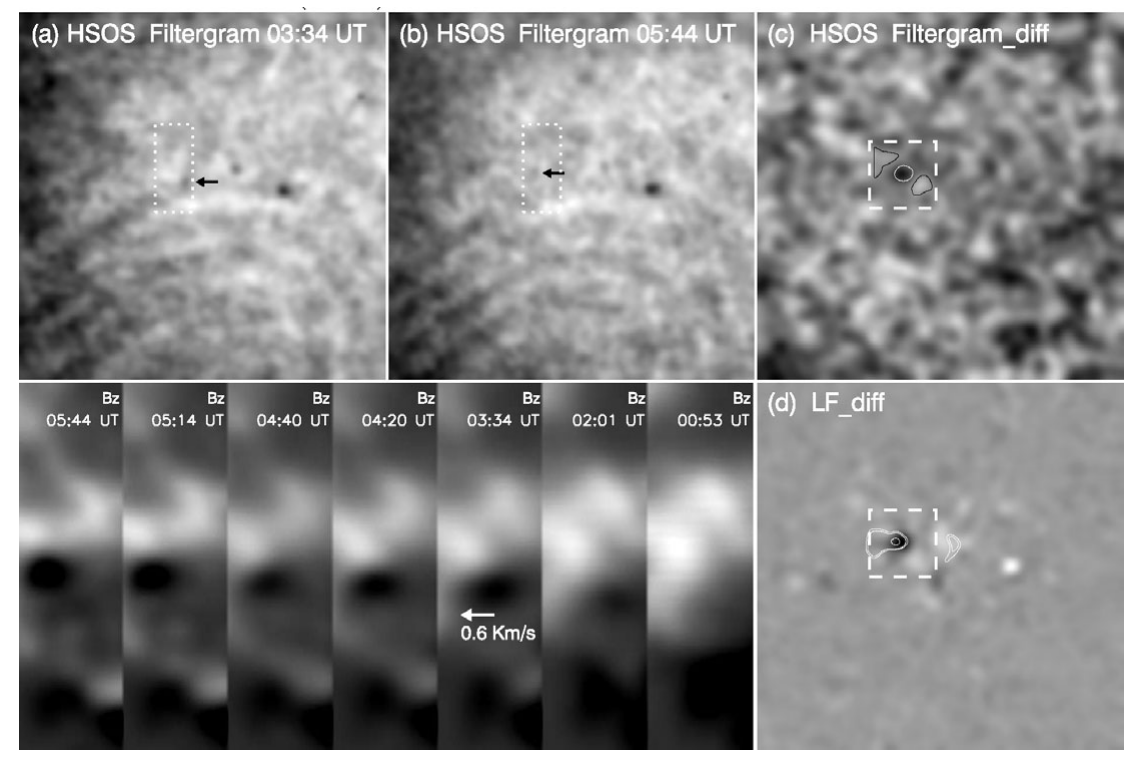

Figure 2. Upper-left moving of a tiny black feature marked by black arrow. (a) and (b) are the pre-flare and post-flare filtergrams of HSOS in FeI $5324.19 \AA$, and (c) and (d) are the WL difference image and net LF map obtained, respectively. The FOV is the same as that of Figure 1. The bottom panels just below panels (a) and (b) are a time sequence of HSOS longitudinal magnetograms showing the upper-left shift of a negative polarity patch with a speed of $0.6 \mathrm{~km}$ $\mathrm{s}^{-1}$, whose FOV is $10.5 \times 35$ arcsec shown in the dotted rectangles of (a) and (b). The contours in (c) are $40 \%$ of the maximal and minimal intensities in the dashed rectangle of (c) and those in (d) are the flare profiles as in Figure 1.

Implied by the changes of vector magnetic fields during a flare, there would be the perturbed Lorentz force reacting on the solar photosphere and solar interior (Hudson et al. (2008)). The net Lorentz force (LF) induced by the magnetic field changes in the WL enhanced area acting on the photosphere, $\delta f_{z}=f_{z 2}-f_{z 1}=\left(B_{z}^{2}-B_{z}^{2}\right)-\left(B_{t}^{2}-B_{t}^{2}\right)$, was downward, where subscripts 1 and 2 refer to pre- and post- flare states, respectively. A 2-D map of the net LF is shown in panel (d) of Figure 2. Evidently, in the region near the flaring PIL, the black (downward) patch in the 2-D LF map seemed to be well consistent with the enhance area in panel (c) of Figure 2. The mean value of the downward $\mathrm{LF}$ in the white rectangle is found to be $-1600 \mathrm{G}^{2}$, and the total integrating area is $2 \times 10^{18} \mathrm{~cm}^{2}$. Therefore, the total net LF is $-3.2 \times 10^{21}$ dynes, which is comparable to the downward LF induced by X-class flare (Wang \& Liu (2010)).

\section{Acknowledgement}

This work is supported by the Grants: KJCX2-EW-T07, 2011CB811401, 11178005, 10878004, 11221063, 11203036, and the Specialized Research Fund for State Key Laboratories.

\section{References}

Ambastha, A., Hagyard, M. J., \& West, E. A. 1993, Solar Phys., 148, 277

Deng, N., Liu, C., Yang, G., Wang, H., \& Denker, C. 2005, ApJ, 623, 1195

Hudson, H. S., Fisher, G. H., \& Welsch, B. T. 2008, in ASP Conf. Ser. 383, Subsurface and Atmospheric Influences on Solar Activity, ed. R. Howe et al. (San Francisco, CA: ASP), p221 
Kosovichev, A. G. \& Zharkova, V. V. 2001, Ap. Lett., 550, 105

Li, H., Sakurai, T., Ichimoto, K., \& UeNo, S. 2000, PASJ, 52, 465

Liu, C., Deng, N., Liu, Y., Falconer, D., Goode, P. R., Denker, C., \& Wang, H. 2005, ApJ, 622, 722

Spirock, T. J., Yurchyshyn, V. B., \& Wang, H. 2002, ApJ, 572, 1072

Sudol, J. J. \& Harvey, J. W. 2005, ApJ, 635, 647

Wang, H. 1992, Solar Phys., 140, 85

Wang, H. \& Liu, C. 2010, Ap. Lett., 716, 195 